\title{
APUNTES SOBRE LOS EFECTOS DE LOS INCENDIOS FORESTALES Y RESTAURACIÓN AMBIENTAL DE ÁREAS QUEMADAS. ESTADO DE LA CUESTIÓN Y PRINCIPIOS GENERALES
}

\author{
F. Pérez-Cabello, M. Echeverría, J. de la Riva y P. Ibarra \\ Dpto. de Geografía y Ordenación del Territorio, Universidad de Zaragoza \\ fcabello@unizar.es
}

\begin{abstract}
Resumen: La incidencia de los incendios forestales en los ecosistemas mediterráneos, como consecuencia del incremento del número de fuegos y de la superficie quemada, se ha convertido en uno de los problemas ambientales de mayor intensidad en las últimas décadas. Alteraciones en los suelos, en la vegetación y en la fauna, en los procesos morfogenéticos, en los paisajes y en las actividades de la población constituyen las principales consecuencias del fuego. Los espacios quemados demandan, tras la perturbación ocurrida, un proceso de restauración apoyado en un análisis integrado del territorio, tanto en sus aspectos físicos, humanos, como especialmente en la interrelación de ambos. En el presente artículo se aborda un breve estado de la cuestión y se presentan algunos principios generales respecto de los que existe consenso en la bibliografía especializada.
\end{abstract}

Palabras clave: Fuegos, consecuencias ambientales, análisis del territorio, plan integral de restauración.

\begin{abstract}
The incidence of wildfires on Mediterranean ecosystems -as a result of the increase of the number of fires and the burned area- has become one of the most important environmental problems in the recent decades. Fire affects soil characteristics, vegetation, wildlife, morphogenetic processes, landscapes and human activities. Once the disturbance occurred, the burned areas require restoration strategies supported by an integrated analysis of the territory in terms of physical and human aspects, and especially in the interaction of them. This paper
\end{abstract}

Recibido: 2-11-10. Aceptado: 2-2-12. 
presents a brief state of the art and reviews some general principles concerning integral restoration of burned areas for which there is consensus in the literature.

Keywords: Wildland fires, environmental effects, analysis of the territory, integral restoration plan.

\section{Introducción}

En la cuenca mediterránea el fuego se ha presentado a lo largo de la historia como un elemento natural. Sin embargo, en las últimas décadas su recurrencia y magnitud están alterando profundamente los ecosistemas forestales. Aunque la acción del fuego afecta al sistema suelo-vegetación-fauna, el efecto de la combustión más inmediato y de mayor envergadura ecológico-paisajística es la destrucción de la vegetación, el componente del medio más vulnerable ante este tipo de perturbaciones y el mejor indicador del grado de afección del resto de componentes del sistema. El efecto más directo del incendio es el chamuscado, que consiste tanto en la quema directa como en la deshidratación de los tejidos a causa de las elevadas temperaturas del fuego. El consiguiente debilitamiento de la vegetación, variable que según las circunstancias e individuos, suele traducirse en retraso del crecimiento y una mayor exposición a los ataques de hongos e insectos. Desde el punto de vista fisionómico-florístico, la bibliografía referida a los efectos del fuego sobre la vegetación es muy extensa. En los ecosistemas mediterráneos, tras un incendio se desencadenan intensos mecanismos de rebrote/germinación de especies y semillas preexistentes que conllevan un rápido recubrimiento del suelo. Este restablecimiento de las comunidades quemadas -"autosucesión"- es un proceso especial, ya que el punto de partida no es cero, al destruirse sólo parte del contenido biológico y conservarse ciertas condiciones edáficas.

Desde el punto de vista de la erosión, la pérdida del dosel vegetal supone la alteración del ciclo hidrológico al modificarse las condiciones evapo-transpiratorias y los parámetros controladores de la escorrentía y de la infiltración. La disminución de la cubierta vegetal implica que la lluvia encuentre menos obstáculos en su trayectoria hacia el suelo, aumentando su energía cinética y, por consiguiente, su capacidad para destruir los agregados edáficos. La desarticulación de la estructura del suelo conlleva una disminución de la porosidad por la formación de costras, responsables en gran parte de la escasa eficacia de la infiltración de agua en el suelo y del aumento de la arroyada superficial (Cerdà, 1995).

En la actualidad, los conocimientos científico-técnicos apuntan a un aumento de las tasas de erosión tras el fuego durante unos meses o años, para luego reducirse y 
volver a la situación previa al incendio (Díaz-Fierros et al., 1982; Cerdà, 1998; Gimeno et al., 2000; Lasanta y Cerdà, 2005; Cerdà y Lasanta, 2005). Este periodo, denominado "de relajación", varía según los escenarios; en algunos casos, el incendio no produce alteraciones significativas y la recuperación es rápida (Pérez-Cabello, 2002; PérezCabello et al., 2000) e, incluso, la alta variabilidad de las lluvias puede provocar que las tasas de erosión durante el año post-incendio desciendan (Sánchez et al., 1994). Sin embargo, también se ha comprobado cómo, en ocasiones, las tasas de erosión altas son persistentes durante años, lo que puede inducir a procesos de degradación de suelos (Bautista, 1999; Andreu et al., 2000). Este periodo de relajación depende, entre otros factores, del manejo que se realice tras el incendio, de las condiciones previas, de la intensidad del fuego, de las precipitaciones posteriores, de la humedad, de la temperatura, etc. Factor clave para entender la recuperación es, pues, el clima; en condiciones de estrés hídrico y escasa presencia de nutrientes la recuperación suele ser más lenta, mientras que en zonas húmedas, con suelos más potentes, el tiempo de relajación es menor.

Otro efecto de los incendios, relacionado directamente con las alteraciones de los parámetros de infiltración y escorrentía, es la formación de sustancias hidrofóbicas en el suelo derivadas de la descomposición de la materia orgánica (Giovannini, 1994), cuya consecuencia es la reducción de la capacidad de infiltración del agua en el suelo. En relación con el contenido de materia orgánica, numerosos autores han detectado descensos importantes tras el fuego (Doerr et al., 2000). Las variaciones en las pérdidas de materia orgánica están en función de los niveles de intensidad registrados. La combustión de la materia orgánica conlleva una importante liberación de nutrientes fácilmente disponibles para el crecimiento de las plantas (cationes solubles, NH4, $\pm \mathrm{N}$, etc.) (Giovannini et al. 1990), aunque la adición de cenizas al suelo no parece estimular el crecimiento vegetal (Tárrega y Luis-Calabuig, 1992). Además, la pérdida de nutrientes como consecuencia de la reactivación morfogenética provoca el consiguiente empobrecimiento del suelo que dificulta el desarrollo de la vegetación e interrumpe y ralentiza los procesos edafogenéticos.

\section{Consecuencias del fuego: estado de la cuestión}

En relación con las consecuencias del fuego existe una extensa bibliografía y un amplio abanico de equipos de investigación, internacionales y nacionales, que tienen en los efectos del fuego y en la detección de zonas quemadas su principal objetivo. Los antecedentes en relación con los efectos del fuego en los ecosistemas se encuentran en EEUU en los años 40 (Anderson, 1949). En la segunda mitad del S. XX y en el ámbito internacional se desarrollan investigaciones en torno al fuego en las cinco regiones del mundo donde el clima mediterráneo propicia su recurrencia: Chile, California, Sudáfrica, Australia y la Cuenca Mediterránea. Entre ellas se deben desta- 
car las relacionadas con los efectos sobre los suelos (Giovannini y Lucchesi, 1983; Kutiel y Naveh, 1987; DeBano et al., 1979; Thomas et al., 1999); la alteración de la cubierta vegetal (Naveh, 1974; Trabaud, 1987; Rego et al., 1991); los cambios en la respuesta hidrológica del suelo en laderas y cuencas de drenaje (Imeson et al., 1992; Scott y van Wyk, 1990; Robichaud y Waldrop, 1994; Moody y Martin, 2001b); las modificaciones del comportamiento erosivo (Prosser, 1990; Wright et al., 1982; Scott, 1993) y el tiempo de relajación de las tasas de erosión y de escorrentía (Helvey, 1980; Inbar et al., 1998; Moody y Martín, 2001a). En Australia y California el estudio de la repelencia de los suelos al agua ha sido el tema más tratado (DeBano et al., 1970; Brown, 1972; Evans et al., 1999), junto a la pérdida de suelo en suspensión (White et al., 1973; Wright et al., 1978) y la exportación de solutos (DeBano y Conrad, 1978). La alteración de la respuesta hidrológica ha sido estudiada en el Oeste de los EEUU (Moody y Martín, 2001b) y en Sudáfrica (Scott y van Wik, 1990). En Israel, los estudios más importantes se han dedicado a las alteraciones de la vegetación, los cambios en la dinámica erosiva y la exportación de solutos (Lavee et al., 1995; Kutiel y Naveh, 1987b).

En el ámbito nacional destaca la presencia de grupos que durante las últimas dos décadas han basado su investigación en el análisis de los efectos del fuego sobre los procesos de erosión. Los estudios en Galicia han sido llevados a cabo desde la Universidad de Santiago por el grupo de Díaz-Fierros, desde el Centro de Investigación Forestal de Lourizán por el grupo de Vega o desde el Departamento de Bioquímica del Suelo (CSIC) por el de Carballas. Otro grupo relevante se ubica en Cataluña, donde Sala, Marqués y Úbeda, entre otros, desde la Universidad de Barcelona han estudiado la dinámica de la erosión tras el fuego y la influencia de los usos del suelo y su manejo; también se ha comprobado cómo la recuperación postincendio es rápida, posiblemente debido a condiciones climáticas húmedas que favorecen la revegetación. A caballo entre la Universidad de Barcelona y el Centro de Estudios Ambientales del Mediterráneo (CEAM) en Valencia, el grupo de Vallejo ha puesto en marcha innumerables trabajos relacionados con el fuego y los usos de suelo, la vegetación tras el incendio.... Otro grupo de investigación se forma en torno a Rubio en el Centro de Investigaciones sobre Desertificación del CSIC en Valencia; los estudios realizados en zonas más áridas han demostrado a este grupo que la recuperación de las condiciones previas es compleja y, parece, que más lenta que en los casos anteriores. También en la Comunidad Valenciana, Cerdà y Mataix aglutinan experiencias en torno a trabajos diversos en relación con incendios y procesos edáficos y erosivos. Y, finalmente, desde la Universidad de Alicante, se ha estudiado el efecto del fuego en pinares y matorrales.

En Aragón, en los últimos 15 años el equipo de Badía (Badía y Martí, 2000; Badía et al., 2010) ha abordado la respuesta de la erosión al fuego en suelos sobre distintas litologías, bajo diferentes cubiertas vegetales y aplicando diferentes tratamientos 
postincendio. El grupo de investigación GEOFOREST -Procesos Geoambientales en Espacios Forestales (http://geoforest.unizar.es/)- de la Universidad de Zaragoza integrado en el grupo Geomorfología y Cambio Global y en el Instituto Universitario de Ciencias Ambientales de Aragón (IUCA)- aborda la cuestión de los fuegos forestales desde dos vertientes temporales: el modelado del riesgo de incendio mediante el análisis de factores físicos y socioeconómicos y el análisis y predicción de los efectos hidro-geomorfológicos sobre el tándem vegetación-suelo, utilizando técnicas experimentales e información procedente de la teledetección (Pérez-Cabello, 2002; PérezCabello et al., 2000, 2006).

La creación de Fuegored (http://grupo.us.es/fuegored/), por parte de investigadores que trabajan en la temática de suelos de incendios, ha supuesto la publicación de varias obras científicas que abordan las múltiples perspectivas del tema y sintetizan la actividad de los principales grupos españoles: Cerdà y Mataix (Eds. 2009), Cerdà y Jordán (Eds. 2010); revista Flamma; Díaz-Raviña et al. (2010).

\section{La gestión post-incendio: experiencias y reflexiones}

Desde el punto de vista de la gestión post-incendio, tras la extinción surgen incógnitas en relación con la aplicación de medidas destinadas a la recuperación integral de los ecosistemas. Aunque existen numerosos estudios en los que se analizan las consecuencias del fuego (Robichaud et al., 2005; Cerdà y Robichaud, 2008; DíazRaviña et al., 2010), el hecho de que sus efectos no sean fácilmente generalizables dificulta la selección de medidas para minimizar las consecuencias negativas. La diferente capacidad natural de respuesta del medio y las interacciones que se producen entre las variables ambientales y los parámetros específicos del fuego (intensidadseveridad) explican, en gran medida, la falta de uniformidad en relación con este último extremo y la necesidad de diseñar programas de gestión específicos para cada zona. En cualquier caso, la restauración es un proceso lento y que requiere una evaluación científica y seguimiento a medio y largo plazo (Pausas y Vallejo, 2008).

Algunas iniciativas de restauración tras el fuego subrayan la necesidad de no realizar actuaciones sin un estudio completo del medio físico y socioeconómico, de sus recursos y de su evolución dinámica, y sin una planificación, en el espacio y en el tiempo, de todas las actuaciones. En este marco, el análisis de la vulnerabilidad del sistema -entendiendo por tal el grado de susceptibilidad al deterioro ante la incidencia de determinadas actuaciones, o el inverso de la capacidad de absorción de posibles alteraciones sin pérdida de calidad- es fundamental en la definición de los planes de restauración. Así, la vulnerabilidad ecológica tras un tipo de perturbación específica en este caso, la irrupción violenta del fuego, hace referencia al grado de potencialidad estructural de las comunidades forestales para recomponer las condiciones y las interrelaciones de los elementos que las conformaban. 
Desde la óptica geomorfológica la capacidad de recuperación se valora en relación con la aptitud del medio para mantener los niveles de actividad edafo-morfológica previos al fuego. Los medios que registran incrementos considerables en la incidencia espacial de las manifestaciones erosivas y experimentan procesos destacados de degradación de los parámetros edáficos son los menos resistentes y con menor capacidad de recuperación. Las precipitaciones en los meses siguientes al incendio pueden provocar importantes problemas de crecidas en los cauces que drenan la zona, un incremento de los riesgos de erosión laminar y en regueros. El papel desempeñado por la severidad sigue planteando algunas dudas -continúa siendo una línea de investigación abierta- pero, en términos generales, la alta severidad se asocia, entre otros procesos, a una mayor calcinación de la cubierta vegetal, un mayor incremento de la hidrofobicidad del suelo y de la erosión (DeBano et al., 1970; Robichaud et al., 2005).

Desde la vertiente de la vegetación, la capacidad de reconstrucción se estima en relación con la aptitud del sistema para lograr los niveles de recubrimiento vegetal y las propiedades fisionómicas y florísticas previas al fuego. De este modo, los sistemas con mayor capacidad son aquellos que presentan una menor divergencia con las condiciones previas al fuego.

El concepto de severidad es fundamental para explicar los efectos del fuego. A pesar de sus múltiples definiciones, la mayoría de los investigadores convienen que la severidad es una medida de la cantidad de materia orgánica perdida por el incendio, la desaparición de la cubierta vegetal y la volatilización o transformación de los componentes del suelo en formas minerales solubles (Miller y Yool, 2002). Por su parte, el concepto intensidad hace referencia al estado del fuego durante la combustión y se mide en términos de temperatura, altura de la llama, cantidad total y tamaño del combustible consumido y transferencia de calor a los estratos superiores (Miller y Yool, 2002). Por tanto, severidad e intensidad son conceptos diferentes, aunque relacionados: la misma intensidad del fuego puede producir diferentes grados de severidad dependiendo de factores como la composición y estructura de la comunidad vegetal previa (Key y Benson, 2005; van Wagtendonk et al., 2004).

La cartografía de la severidad sirve para identificar áreas problemáticas y así ayudar a los gestores en la localización de recursos y esfuerzos de restauración, disminuyendo el tiempo y costes económicos al reducir la necesidad de un trabajo de campo a gran escala (Patterson y Yool, 1998). En este marco, la teledetección es una herramienta muy adecuada, como también lo es para estudiar los procesos postfuego, ya que los efectos del fuego sobre la vegetación y el suelo generan cambios en el comportamiento espectral de estas cubiertas (Díaz-Delgado y Pons, 2001; Riaño et al., 2002). 
Existen diferentes propuestas sobre mediciones de la severidad en el campo (Patterson y Yool, 1998) destacando el Fire Effects Monitoring and Inventory Project (FIREMON) por tratarse de un inventario nacional y por estar bien adaptado a la teledetección. Este proyecto desarrolló el Composite Burn Index (CBI), profusamente utilizado, que aporta como novedad una medida cuantitativa de la severidad del fuego, facilitando su puesta en relación con los datos de las imágenes de satélite (Key y Benson, 2005). Los valores del índice CBI han sido utilizados como información para validar los valores de severidad obtenidos mediante teledetección (van Wagtendonk et al., 2004) y también como instrumento de evaluación de índices espectrales.

\section{Las tecnologías de la información geográfica en el análisis de los incendios}

La teledetección y los SIG ofrecen posibilidades muy interesantes en relación con la detección y seguimiento de focos activos, con la evaluación de daños y niveles de severidad y con la cartografía y el seguimiento multitemporal de la recuperación de superficies quemadas, aportando nuevas perspectivas espacio-temporales (van Wagtendok et al., 2004). Esta potencialidad se fundamenta, con frecuencia, en las importantes mejoras que reportan al seguimiento y control de la vegetación frente a las técnicas más tradicionales; en este sentido, los SIG aportan la operatividad y eficacia de sus funcionalidades en el contexto de los análisis territoriales, facilitados por la compatibilidad de las estructuras digitales y los formatos de intercambio (Salas y Chuvieco, 1994).

Desde los años 70 se han venido empleando imágenes de satélite para la detección de zonas quemadas, utilizando casi de manera generalizada sensores de alta y media resolución espacial (Landsat TM, SPOT-HRV). En nuestro país la primera experiencia se desarrolló en Cataluña con el Proyecto Piloto 1984 de la European Space Agency (ESA). Chuvieco y Congalton (1988) evaluaron las superficies afectadas por incendios mediante un proceso de clasificación mixto visual/digital en la costa mediterránea. El Centre de Recerca Ecològica i Aplicacions Forestals de Catalunya (CREAF) desarrolló una metodología semiautomática para la evaluación de superficies quemadas a partir de una serie temporal de imágenes Landsat MSS, con el propósito de cartografiar la recurrencia del fuego, facilitar el análisis estadístico de los patrones espacio-temporales de ocurrencia y el seguimiento de la regeneración a través de índices de vegetación (Díaz-Delgado et al., 1999); también destaca el grupo de Meliá de la Universidad de Valencia (Viedma et al., 1997).

A nivel internacional, y resopecto a la severidad, destaca el National Burn Severity Mapping Proyect (2001), con investigadores del United States Geological Survey (USGS) y del Nacional Park Service (NPS), dentro del Joint Fire Science Program, para 
la estimación de parámetros de severidad utilizando el Normalized Burn Ratio (NBR) y datos de campo.

En lo referente a los efectos de los incendios y el diseño de medidas correctoras de restauración, existen diversos grupos de trabajo; en la bibliografía especializada se pueden encontrar experiencias concretas aplicadas en los últimos años. Algunos de estos equipos y trabajos son los siguientes:

- Los programas de I+D sobre prevención de incendios y restauración de montes quemados de la Fundación Centro de Estudios Ambientales del Mediterráneo (CEAM). Recientemente se ha publicado un volumen específico sobre efectos del fuego sobre el suelo y estrategias de restauración en la serie Land Reconstruction and Management (Cerdà y Robichaud, Eds. 2008), que recoge las principales experiencias en el ámbito internacional.

- La actividad desarrollada en torno a la definición de bases ecológicas para la restauración preventiva de zonas quemadas en el Centro de Investigación e Información Ambiental de Galicia-Lourizán, Departamento de Protección Ambiental.

- Los informes de los grupos de trabajo respectivos de "Incendios Forestales" y "Selvicultura" del VI y VII Congreso Nacional de Medio Ambiente (CONAMA, 2002, 2006).

- El número 6 de la Serie "Manuales de Desarrollo sostenible" de la Fundación Banco Santander, titulado "Criterios de restauración de Zonas incendiadas".

- La red temática "Efectos de los incendios forestales sobre los suelos, FUEGORED“ (http://grupo.us.es/fuegored/).

- Así mismo, otras referencias bibliográficas especialmente interesantes en esta misma línea son las de Copano y Díaz (2001), García Alonso y Copano (2002, 2008), TRAGSATEC (2005), WWF/ADENA (2006).

\section{Claves ecológicas para la restauración del bosque mediterráneo}

La restauración ecológica, recuperación de componentes y funciones de ecosistemas degradados, se basa en principios y experiencias de la Ecología, tales como la sucesión ecológica y, en especial, la sucesión secundaria, relacionada con la dinámica de ambientes que han sido desviados natural o artificialmente de la sucesión original o primaria. Una de las conclusiones más generalizadas del estudio de la sucesión secundaria es que, en el proceso de recuperación de ambientes degradados nunca se repite exactamente la trayectoria de la sucesión original previa a la perturbación (Trabaud, 1998, Pausas y Vallejo, 1999). 
Las poblaciones de las distintas especies deben seguir su evolución en un escenario ecológico en el que ellas son actores y dan expresión a su mensaje genético reajustándose a los factores ambientales. Por ello, es esencial en todo proyecto de restauración ecológica definir con suficiente claridad y con la oportuna precisión los objetivos y hacer un seguimiento de los resultados para comprobar su cumplimiento, reajustando las actuaciones si es necesario. Aún con esto no se debe esperar que un proyecto de restauración ambiental transcurra sin problemas o que esté bien perfilado. Las diversas etapas que se suceden en su realización son otros tantos eslabones por los cuales puede fallar radicalmente el proyecto, o rebajarse su grado de éxito.

Frente a las clásicas actuaciones cerradas y ceñidas a un proyecto de obras, la solución pasa por establecer varias fases -a modo de proyectos concatenados- que abarquen los distintos estadios de realización del proyecto (Pausas y Vallejo, 2008). Será preciso garantizar la conexión, la responsabilidad y la continuidad entre estas etapas, lo que exigirá, quizás, el establecimiento por parte de la Administración de un grupo de seguimiento de este proyecto de restauración integral.

\subsection{Principios generales de la restauración ecológica}

La restauración ecológica trata de devolver el ecosistema perturbado a un estado lo más parecido a su condición previa al fuego. Ello exige reparar el ecosistema degradado, reconstruyendo las estructuras y funciones perdidas. No se trata sólo de recuperar especies, como si de un vivero se tratara, sino de restaurar las interacciones y procesos ecológicos en los que dichas especies se relacionan entre sí y con el medio abiótico. No se trata de recuperar escenarios físicos, sino también los organismos que protagonizan la función en el teatro ecológico. No se trata de crear comunidades artificiales, ni de plantar jardines que requieran cuidados frecuentes, se trata de generar sistemas que funcionen de acuerdo con los principios ecológicos, capaces de automantenerse e integrarse en su contexto e incluso de madurar por sí solos. Las soluciones tecnológicas deben estar al servicio de la Ecología.

En la práctica, un proyecto de restauración ecológica necesita de:

- Diagnóstico de la situación del ecosistema degradado.

- Definición del ecosistema hacia el que se pretende reconducirlo. La diferencia entre la situación actual y la meta de referencia expresará la magnitud e intensidad del esfuerzo de restauración.

- Proyecto de restauración en sí, con la planificación espacio-temporal de las actuaciones, que deben favorecer los mecanismos naturales de recuperación, acelerando el proceso de sucesión. 
Una restauración integral sólo se consigue tras un período de tiempo en ocasiones considerable, debiendo las actuaciones ir acompañadas de una campaña de información, comunicación y educación ambiental para que la sociedad comprenda las razones de las mismas. Los tiempos deben ser respetados, supeditando las actuaciones realizadas a corto plazo a los objetivos finales. Se trata de recuperar calidad, diversidad y funcionalidad a nivel ecológico, genético y paisajístico; para ello, las labores de restauración necesitan muchas veces de trabajos artesanales mantenidos en el tiempo, más que de maquinaria pesada. Así, por ejemplo, no se logra restaurar un bosque mediterráneo -con su gran diversidad de especies y su considerable heterogeneidad espacial- con una sola plantación masiva y simultánea de unas pocas especies en un marco de plantación homogéneo; hay que empezar colocando plantones y semillas sólo en los microhábitats que pueden actuar como nichos de regeneración efectivos (por ejemplo bajo matorrales pioneros), evitando los micro-hábitats muy expuestos al Sol, donde la mortalidad estival es muy elevada.

El inicio de la sucesión primaria en terrenos muy degradados pasa por mejorar las propiedades del suelo que permitan un posterior establecimiento de la cubierta vegetal. Igualmente, la recuperación de la vegetación característica de los bosques y matorrales mediterráneos está íntimamente asociada a la actividad de los animales mutualistas -aves y mamíferos- que dispersan las semillas de la mayoría de las especies leñosas. También se puede determinar qué tipo de actividad humana es compatible o no con una determinada etapa del proyecto. Por ejemplo, la presión ganadera sobre la vegetación leñosa debe ser muy baja cuando dicha vegetación aparece en forma de plántulas, juveniles y rebrotes de leñosas; pero esa misma formación vegetal admite una carga ganadera más elevada cuando las copas sobrepasan determinada altura que las libre del bocado del ungulado.

Tan necesaria como una buena planificación temporal de las actuaciones son las labores de seguimiento y control durante y después de la restauración, comparando los resultados obtenidos con otros ecosistemas naturales de referencia. Ello requiere marcos temporales de seguimiento superiores a los 5-10 años.

Tanto en la elaboración de los proyectos, como durante su ejecución y seguimiento los trabajos de restauración deben ser lugar de encuentro entre profesionales de distinta formación. Una buena restauración ecológica debe incluir también aspectos históricos, sociales, culturales y estéticos, entre otros. Es por ello un terreno fértil donde ingenieros, geógrafos, ecólogos, edafólogos, etc. pueden trabajar en torno a una planificación ambiental integral, con una definición y priorización clara de los objetivos. 


\section{Bibliografía}

Anderson, H.W. (1949). Does burning increase surface runoff? Journal of Forestry, 47, 54-57.

Andreu, V., Imeson, A.C. y Rubio, J.L. (2000). Temporal changes in soil aggregates and water erosion after a wildfire in a Mediterranean pine forest. Catena, 44, 6944.

Badía, D. y Martí, C. (2000). Seeding and mulching treatments as conservation mesures of two burned soils in the Central Ebro Valley, NE Spain. Arid Soil Research and Rehabilitation, 13, 219-232.

Badía, D., Martí, C., León, F.J, Ibarra, P. y Echeverría, M.T. (2010). Soil toposequence (Xerolls) in the Montes de Zuera (Zaragoza, Spain). En Actas del Congreso Iberoamericano de la Ciencia del Suelo, Granada.

Bautista, S. (1999). Regeneración post-incendio de un pinar (Pinus halepensis, Miller) en ambiente semiárido. Erosión del suelo y medidas de conservación a corto plazo. Tesis Doctoral, Facultad de Ciencias, Universidad de Alicante, 238 pp.

Brown, J.H.A. (1972). Hydrological effects of a Bushfire in a catchment in South-Eastern New South Wales. Journal of Hydrology, 15, 77-96.

Cerdà, A. (1995). Surface hydrology of three burnt soils, south-east Spain. En Fantechi, R. Balabanis, P.D. y Rubio, J.L. (eds) Desertification in a European context. Physical and socio-economic aspects, Bruselas, European Commission, pp. 437445.

Cerdà, A. (1998). Postfire dynamics of erosional processes under mediterranean climatic conditions. Z. für Geomorph., 42, 373-398.
Cerdà, A. y Jordán, A. (Eds. 2010). Actualización en métodos y técnicas para el estudio de los suelos afectados por incendios forestales. Valencia, Cátedra de Divulgación de la Ciencia de la Universidad de Valencia, 521 pp.

Cerdà, A. y Lasanta, T. (2005). Long-term erosional responses after fire in the Central Spanish Pyrenees. 1. Water and sediment yield. Catena, 60(1), 58-80.

Cerdà, A. y Mataix-Solera, J. (Eds. 2009). Efectos de los incendios forestales sobre los suelos en España, Valencia, Cátedra de Divulgación de la Ciencia de la Universidad de Valencia, 529 pp.

Cerdà, A. y Robichaud, P. (Eds. 2008). Fire Effects on Soils and Restoration strategies, USA, Science Publisers, 589 pp.

CONAMA (2002). Selvicultura: una necesidad para la conservación de nuestros montes y la prevención de los incendios. Grupo de Trabajo 2, VI Congreso Nacional de Medio Ambiente. Madrid.

CONAMA (2006). Incendios forestales: tratamiento de superficies quemadas. Grupo de Trabajo 6, VIII Congreso Nacional de Medio Ambiente. Madrid.

Copano, C. y Díaz, M. (2001). Proyecto de Demostración de Lucha contra la Desertificación. Regeneración y Plan de Manejo de Áreas Semáridas Incendiadas en la Sierra de Ricote, Monte $\mathrm{n}^{\circ} \mathrm{o} 25 \mathrm{del}$ C.U.P., T.M. de Ricote, Murcia, MMA.

DeBano, L.F. y Conrad, C.E. (1978). The effect of fire on nutrients in a chaparral ecosystem. Ecology, 59, 489-497.

DeBano, L.F., Eberlein, G.E. y Dunn, P.H. 1979. Effects of burning on chaparral soils. I. Soil nitrogen. Soil Sci. Soc. Amer. Journal, 43, 504-509. 
DeBano, L.F., Mann, L.D. y Hamilton, D.A (1970). Translocation of hydrophobic Substances into Soil by Burning Organic Litter. Soil Sci. Soc. Amer. Journal 34, 130 133.

Díaz-Delgado, R. y Pons, X. (2001). Spatial patterns of forest fires in Catalonia (NE of Spain) along the period 1975-1995 analysis of vegetation recovery after the fire. Forest Ecology and Management, 147, 67-74.

Díaz-Fierros, F., Gil, V., Cabaneiro, A. Carballas, T., Leiros de la Peña, M.C. y Villar, M.C. (1982). Efectos erosivos de los incendios forestales en suelos de Galicia. Anales de Edafología y Agrobiología XLI, 627-639.

Díaz-Raviña, M., Benito, E., Carballas, T., Fortúrbel, T. y Vega, J.A. (Eds. 2010). Investigación y gestión para la protección del suelo y restauración de los ecosistemas forestales afectados por incendios forestales. Santiago de Compostela, CSIC Fuegored 2010, 326 pp

Doerr, S.H., Shakesby, R.A. y Walsh, R.P.D (2000). Soil water repellency: its causes, characteristics and hydro-geomorphological significance. Earth Sciences Reviews, 51, 33-35.

Evans, K.G., Saynor, M.J. y Willgoose, G.R. (1999). Changes in hydrology, sediment loss and microtopography of a vegetated mine waste rock dump impacted by fire. Land Degradation and Development, 10, 507-522.

García-Alonso, M.T. y Copano-González de Heredia, C. (2002). Restauración hidrológico-forestal para el control de la erosión de la zona afectada por el incendio de julio de 1994 en la comarca de "Els Ports" (Castellón).

Gimeno, E., Andreu, V. y Rubio, J.C. (2000). Changes in organic matter, nitrogen, phos- phorus and cations in soil as a result of fire and water erosion in a Mediterranean landscape. European Journal of Soil Science 51, 201-210.

Giovannini, G. (1994). The effect of fire on soil quality. En Sala, M. y Rubio, J.L. (eds) Soil erosion as a consequence of forest fire, Logroño, Geoforma Ediciones, pp. 15-27.

Giovannini, G. y Lucchesi, S. (1983). Effect of fire on hydrophobic and cementing substances of soil aggregates. Soil Science, $136,231-36$

Giovannini, G., Lucchesi, S. y Giachetti, M. (1990). Effects of heating on some chemical parameters related to soil fertility and plant growth. Soil Science, 149(6), 344350.

Imeson, A.C., Verstraten, J.M., van Mulligen, E.J. y Sevink, J. (1992). The Effect of Fire and Water Repellency on Infiltration and Runoff under Mediterranean Type Forest. Catena, 19, 345-361

Inbar, M., Tamir, M. y Wittenberg, L. (1998). Runoff and erosion processes after a forest fire in Mount Carmel, a Mediterranean area. Geomorphology, 24, 17-33.

Key, C.H. y Benson, N.C. (2005). Landscape assessment: ground measure of severity, the Composite Burn Index, and remote sensing of severity, the Normalized Burn Ratio. En Lutes, D.C. (ed) FIREMON: Fire effects monitoring and inventory system, Ogden, Ut, General Technical Report, RMRS-GTR- 164-CD: LA1-LA51, USDA.

Kutiel, P. y Naveh, Z. (1987). The effect of fire on nutrients in a pine forest soil. Plant and Soil, 104, 269-274.

Lasanta, T. y Cerdà, A. (2005). Long-term erosional responses alter FIRE in the Central Spanish Pyrenees. 2. Solute release. Catena, 60(1), 81-100 
Lavee, H., Kutiel, P., Segev, M. y Benyamini, Y. (1995). Effect of surface roughness on runoff and erosion in an Mediterranean ecosystem: the role of Fire. Geomorphology, 11, 227-234.

Miller, J.D. Y Yool, S.R. (2002). Mapping forest post-fire canopy consumption in several overstory types using multi-temporal Landsat TM and ETM data. Remote Sensing of Environment, 82, 481-496.

Moody J.A. y Martin, D.A. (2001a). Post-fire, rainfall intensity-peak discharge relation for three mountainous watersheds in the western USA. Hydrological processes, 15, 2981-2993.

Moody, J.A. y Martin, D.A. (2001b). Initial hydrologic and geomorphic response following a wildfire in the Colorado Front Range. Earth Surface Processes and Landforms 26, 1049-1070.

Naveh, Z. (1974). Effects of fire in the Mediterranean region. En Koxlowski, T.T. y Ahlgren, C.E. (eds) Fire and ecosystems, New York, Academic Press, pp. 372-390.

Patterson, M. y Yool, S.R. (1998). Mapping fire-induced vegetation mortality using Landsat thematic mapper data: A comparison of linear transformation techniques. Remote Sensing of Environment, 65 , 132-142.

Pausas, J. y Vallejo R. (1999). The role of fire in European Mediterranean ecosystems. En Chuvieco, E. (ed.) Remote Sensing of Large Wildfires in the European Mediterranean Basin, Springer-Verlag, pp. 3-16.

Pausas, J. y Vallejo, R. (2008). Bases ecológicas para convivir con los incendios forestales en la Región mediterránea: decálogo. Ecosistemas 17 (2), 128-129

Pérez-Cabello, F. (2002). Paisajes forestales y fuego en el prepirineo occidental oscense.
Un modelo regional de recontrucción ambiental. Zaragoza, Consejo de Protección de la Naturaleza de Aragón, 349 pp.

Pérez-Cabello, F., Cancer, L., de la Riva, J., Echeverría, MำT. e Ibarra, P. (2000). El papel de la vegetación quemada y del proceso de regeneración vegetal en relación con la pérdida de suelo. El caso del incendio de Agüero (Prepirineo oscense, España). Il Suolo, 3, 24-30.

Pérez-Cabello, F., de la Riva Fernández, J., Montorio Llovería, R. y García-Martín, A. (2006). Mapping erosion-sensitive areas after wildfire using fieldwork, remote sensing and GIS techniques on a regional scale. JGR-Biogeosciences, 111 (G04S10), $13 \mathrm{pp}$.

Prosser, I.P. (1990). Fire, humans and denudation at Wangrah Creek, southern Tablelands. Australian Geographical studies, 28, 77-95.

Rego, F.C., Bunting, S.C. y da Silva, J.M. (1991). Changes in understory vegetation following prescribed fire in maritime pine forests. Forest Ecology and Management, 41, 21-31.

Robichaud, P,R., Beyers, J.L. y Neary, D.G. (2005). Watershed rehabilitation. En Neary, D.G., Ryan, K.C. y DeBano, L.F. (eds) Wildland Fire in Ecosystems. Effects of Fire on Soil and Water, Odgen, UT, USDA, Vol. 4, pp.179-198.

Robichaud, P.R. y Waldrop, T.A. (1994). A comparison of surface runoff and sediment yields form low-and-high severity site preparation burns. Water Research Bulletin, 30, 27-34.

Sala, M. (1988). Slope runoff and sediment production in two mediterranean mountain environments. Catena supplement, 12 , 13-29. 
Salas, F.J. y Chuvieco, E. (1995). Aplicación de imágenes Landsat-TM a la cartografía de modelos combustibles. Revista de Teledetección, 5, 18-28.

Sánchez, J.R., Mangas, V.J., Ortiz, C. y Bellot, J. (1994). Forest fire effect on soil chemical properties and runoff. En Sala, M. y Rubio, J.L. (eds) Soil erosion and degradation as a consequence of forest fires, Logroño, Geoforma Ediciones, pp. 53-65.

Scott, D.F. (1993). The hydrological effects of fire in South African mountain catchments. Journal of Hydrology 150, 409-432.

Scott, D.F. y van Wyk, D.B. (1990). The effects of wildfire on soil wettability and hydrological behaviour of an afforested catchment. Journal of Hydrology, 121, 239-256.

Soler, M. y Sala, M. (1992). Effects of fire and of clearing in a Mediterranean Quercus ilex woodland: an experimental approach Catena, 19, 321-332

Soler, M., Sala, M. y Gallart, F. (1994). Post fire evolution of runoff and erosion during aneighteen month period. En Sala, M. y Rubio, J.L. (eds) Soil erosion and degradation as a consequence of forest fires, Logroño, Geoforma Ediciones, pp. 149161.

Tárrega, R. y Luis-Calabuig, E. (1992). Los Incendios Forestales en León. Conocer León, 10: 16-55.

Thomas, A.D., Walsh, R.P.D. y Shakesby, R.A. (1999). Nutrient losses in eroded sediment alter fire in eucalyptus and pine forests in the wet Mediterranean environment of northern Portugal. Catena, 36, 283-302.
Trabaud, L. (1987). Fire and survival traits of plants. En Trabaud, L. (Ed) The role of fire in ecological systems, The Hague, SPB Acad Publ, pp. 65-89.

Trabaud, L. (1998). Recuperación y regeneración de sistemas mediterráneos incendiados. Incendios Forestales. Serie Geográfica. 7, pp. 37-47.

TRAGSATEC-Junta de Comunidades de Castilla-La Mancha (2005). Plan de actuaciones urgentes en la zona afectada por el incendio forestal de Riba de Saelices.

Vallejo, V.R., Díaz Fierros, F. y de la Rosa, D. (2005). Impactos sobre recursos edáficos. En Evaluación de los impactos del cambio climático en España (ECCE), Madrid, Ministerio de Medio Ambiente, pp. 355-397

van Wagtendonk, J.W., Root, R.R. y Key, C.H. (2004). Comparison of AVIRIS and Landsat ETM+ detection capabilities for burn severity. Remote Sensing of Environment, 92, 397-408

White, E.M., Trompson, W.W. y Gartner, F.R. (1973). Heat effects on nutrient release from soils under ponderosa pine. Journal of Range Management, 26, 22-24

Wright, H.A., Churchill, F.M. y Clarck, W.C. (1978). Influence of prescribed burning on infiltration and sediment production in the pinyon-juniper woodland, Nevada. Journal of Range Management, 31, 250253.

Wright, H.A., Churchill, F.M. y Clarck, W.C. (1982). Soil loss, runoff, and water quality of seeded and unseeded step watersheds following prescribed burning. Journal of Range Management, 35, 382-385. 